\title{
Color Stability of a Pigmented Elastomer for Maxillofacial Appliances*
}

\author{
ANDREW KORAN, ROBERT YU, JOHN M. POWERS, and ROBERT G. CRAIG
}

The University of Michigan, School of Dentistry, Ann Arbor, Michigan 48109

The color stability of a series of eleven maxillofacial pigments was determined after accelerated aging using reflection spectrophotometry. The results indicate that seven of the pigments demonstrated good to excellent color stability, while four of the pigments were less promising for clinical use.

\section{J Dent Res 58(5):1450-1454, May 1979}

\section{Introduction.}

Maxillofacial appliances require frequent replacement because of rapid discoloration in a service environment or degradation of the physical and dynamic properties of the base elastomer or both. Frequent replacement is not within the financial capability of many patients, and the gradual discoloration of an appliance with time may cause embarrassment for a patient with an already-insulted ego.

Sweeney ${ }^{1}$ reported on the use of an accelerated aging chamber in evaluating the color stability of maxillofacial materials. Cantor and his associates ${ }^{2}$ used a reflectance spectrophotometer to evaluate the color of maxillofacial elastomers. By studying the color of human skin and pigmented maxillofacial materials, they suggested that materials and pigments could be blended to match the color of human skin. Goldberg ${ }^{3}$ used a sunlamp and a reflectance spectrophotometer to study the color stability of several maxillofacial materials. Craig and Koran ${ }^{4}$ used an accelerated weathering chamber and a reflectance spectrophotometer to study several maxillofacial mater-

Received for publication May 19, 1978.

Accepted for publication June 30, 1978.

*This investigation was entirely supported by USPHS Research Grant DE-04136 from the National Institute of Dental Research, National Institutes of Health, Bethesda, Maryland 20014.

This investigation was presented at the Annual Meeting of the American Association for Dental Research in Las Vegas, Nevada, June, 1977. ials for color stability. Three of the materials evaluated had excellent color stability, and it was suggested that other factors might be responsible for the discoloration that is seen clinically.

This study measures the color stability of eleven maxillofacial pigments, each incorporated into a maxillofacial elastomer with known color stability under conditions of accelerated aging. This knowledge will be useful in understanding the mechanisms of color degradation in a clinical environment.

\section{Materials and methods.}

A series of 11 maxillofacial pigments* was chosen. They are dry mineral earth pigments and are widely used for pigmenting maxillofacial appliances. The colors were white, yellow, dark buff, medium brown, light brown, red brown, black, red, blue, light orange, and orange yellow. An RTV silicone $e^{t}$ was chosen for pigmentation since it was found to have excellent color stability in a previous study. ${ }^{4}$ The pigments were incorporated into the silicone base at a concentration of 0.2 percent by weight, and the samples were processed according to the manufacturer's instructions. Samples $(3.5 \mathrm{~mm} \times 4 \mathrm{~cm} \times 6 \mathrm{~cm}$ ) were processed in aluminum molds. Three samples were made for each of the 11 pigments.

Curves of percent reflectance versus wavelength $(\lambda)$ were obtained for each sample between 405 and $700 \mathrm{~nm}$ with a doublebeam, ultraviolet-visible spectrophotometer and integrating sphere 8 in the specular

*Artskin Products, Inc.

† Silastic 44210 , base - HHC074, catalyst HHP094, Dow Corning Corporation, Inc., Midland, MI.

+ACTA CIII UV-Visible Spectrophotometer, Beckman Instruments, Inc., Irvine, CA 92664.

$\S$ ASPH-U Integrating Sphere, Beckman Instruments, Inc., Irvine, CA 92664. 
reflection mode. Each specimen was evaluated in the sample port ( $25 \mathrm{~mm}$ in diameter) under two conditions: (1) backed by a black standard $^{\infty}$, and (2) backed by a white standard 9 . A second white standard was used in the reference port for calibration of zero and 100 percent reflectance and to obtain data. Tristimulus values $(X, Y, Z)$ relative to the $1931 \mathrm{CIE}^{* *}$ color-matching functions for CIE standard illuminant A were determined by numerical integration $(\Delta \lambda=$ $5 \mathrm{~nm}$ ) as described elsewhere ${ }^{5}$. Values of CIE chromaticity coordinates $(x, y)$ were calculated from the tristimulus values 5 and were used to obtain dominant wavelength and excitation purity from CIE chromaticity data $(1931)^{5}$ with the use of a computer program*. Luminous reflectance was equal to the tristimulus value, Y. An estimate of the opacity of each sample was obtained by calculation of the contrast ratio ${ }^{6}, \mathrm{Y}_{\text {black }} / \mathrm{Y}_{\text {white }}$.

After measuring the color of each sample after processing, the samples were placed in an accelerated aging chamber $\dagger$ and subjected continuously for 900 hours to the radiation of a 2500 watt xenon light source with borosilicate filters and an intermittent distilled water spray for 18 minutes every 102 minutes. The relative humidity was maintained at 90 percent, and the temperature of the chamber was $43 \mathrm{C}$ or a black panel temperature of $63 \mathrm{C}$.

The color of the samples was measured after 900 hours in the weathering chamber. Means and standard deviations were calculated, and luminous reflectance, contrast ratio, dominant wavelength, and excitation purity were compared for each pigment before and after aging by analysis of variance 7 and Tukey's multiple comparison test ${ }^{8}$ at a 95 percent confidence level.

\section{Results.}

The results for luminous reflectance are

${ }^{\infty}$ Part No. 375287, Beckman Instruments, Inc., Irvine, CA 92664.

I Part No. 104384, Beckman Instruments, Inc., Irvine, CA 92664.

**International Commission on Illumination.

* The computer program is available on request from the authors.

†Weather-Ometer 25-WR, Atlas Electric Devices Co., Chicago, IL 60613.

$\$ 12-2881$ Xenon burner, Atlas Electric Devices Co., Chicago, IL 60613. shown in Table 1. There was no change in luminous reflectance for pigments \#7 (black) and \#8 (red). Several pigments demonstrated very small, but statistically significant, changes in luminous reflectance. This group included pigments \#3 (dark buff), \#4 (medium brown), \#5 (light brown), \#6 (red brown), and \#9 (blue). Pigment \#10 (light orange) showed a moderate change of 2.7 after aging. Three pigments demonstrated very large changes in luminous reflectance. These were $\# 1$ (white) which changed $8.7, \# 2$ (yellow) which changed 15.0 , and \#11 (orange yellow) which changed 12.3. In all instances where there was a change, the luminous reflectance decreased and the samples were darker.

The results for contrast ratio are shown in Table 2. There was no change for contrast ratio before and after aging for six pigments which included \#3 (dark buff), \#4 (medium brown), \#5 (light brown), \#6 (red brown), \#8 (red), and \#9 (blue). Small changes in contrast ratio were observed for pigments \#1 (white), \#7 (black), and \#10 (light orange). Two pigments changed considerably in contrast ratio after aging. These were pigments \#2 (yellow), which decreased by 0.45 , and \#11 (orange yellow), which decreased by 0.43 . Four pigments became more translucent, and one pigment (\#1 white) became more opaque.

The results for dominant wavelength are shown in Table 3 . The following pigments did not change in dominant wavelength: \#2 (yellow), \#3 (dark buff), \#4 (medium brown), \#5 (light brown), \#6 (red brown), and $\# 7$ (black). Four pigments showed small changes in dominant wavelength at 900 hours. These were \#8 (red) with a decrease of $2.4 \mathrm{~nm}$, \#9 (blue) with an increase of $0.7 \mathrm{~nm}, \# 10$ (light orange) with a decrease of $0.6 \mathrm{~nm}$, and $\# 11$ (orange yellow) with a decrease of $2.7 \mathrm{~nm}$. Only one pigment, \#1 (white), changed considerably in dominant wavelength, with an increase of $17.3 \mathrm{~nm}$.

The results for excitation purity are shown in Table 4. Four pigments did not change in excitation purity before and after aging: \#3 (dark buff), \#5 (light brown), \#7 (black), and \#8 (red). Four pigments had small changes in excitation purity at 900 hours. These were \#4 (medium brown) with a change of 0.014 , \#6 (red brown) 
TABLE 1

LUMINOUS REFLECTANCE OF PIGMENTS IN ELASTOMER AT 0 AND 900 HOURS

\begin{tabular}{lccr}
\hline \multicolumn{1}{c}{ Pigment } & 0 Hours & 900 Hours & Change \\
\hline 1 white & $84.9(0.7)^{*}$ & $76.2(0.4)$ & -8.7 \\
2 yellow & $72.8(0.7)$ & $57.8(0.5)$ & -15.0 \\
3 dark buff & $43.7(0.2)$ & $42.8(0.2)$ & -0.9 \\
4 medium brown & $10.2(0.3)$ & $9.8(0.1)$ & -0.4 \\
5 light brown & $30.8(0.2)$ & $30.2(0.2)$ & -0.6 \\
6 red brown & $10.9(0.1)$ & $10.6(0.1)$ & -0.3 \\
7 black & $3.12(0.03)$ & $2.94(0.05)$ & -0.2 \\
8 red & $14.9(0.2)$ & $14.8(0.1)$ & -0.1 \\
9 blue & $9.5(0.3)$ & $9.1(0.2)$ & -0.4 \\
10 light orange & $47.4(0.3)$ & $44.7(0.3)$ & -2.7 \\
11 orange yellow & $71.2(0.8)$ & $58.9(0.3)$ & -12.3 \\
\hline
\end{tabular}

* Mean with standard deviation in parentheses.

Tukey's Interval is 0.2 at the $95 \%$ level of confidence.

Underline indicates no statistical difference.

0.014 change, \#9 (blue) -0.008 change, and \#10 (light orange) -0.024 change. Three pigments had large changes in excitation purity, and these were: \#1 (white) 0.092 , \#2 (yellow) -0.757 , and \#11 (orange yellow) -0.669 . When changes did occur, three pigments increased in excitation purity, while four pigments decreased in excitation purity.

\section{Discussion.}

The combination of color measurements with the reflectance spectrophotometer and accelerated aging was useful in determining which of the pigments were more color stable. Very small changes in luminous reflectance, contrast ratio, dominant wave- length, and excitation purity could be statistically documented.

In all instances where luminous reflectance changed after 900 hours of aging, the pigmented samples were darker, and the change ranged from -0.3 to -15.0 . Changes in luminous reflectance are easily perceived clinically, and when the excitation purity is low, luminous reflectance is the most significant color parameter.

Only five pigments changed in contrast ratio after 900 hours of aging. It was interesting that four pigments became more translucent while one pigment, \#1 (white), became more opaque. Two pigments, \#2 (yellow) and \#11 (orange yellow), had very large decreases in contrast ratio of -0.45 and -0.43 , respectively. Since the base elas-

TABLE 2

CONTRAST RATIO OF PIGMENTS IN ELASTOMER AT 0 AND 900 HOURS

\begin{tabular}{llcr}
\hline \multicolumn{1}{c}{ Pigment } & 0 Hours & 900 Hours & Change \\
\hline 1 white & $0.690(0.006)^{*}$ & $0.717(0.005)$ & 0.027 \\
2 yellow & $0.706(0.008)$ & $0.256(0.011)$ & -0.450 \\
3 dark buff & $0.830(0.018)$ & $0.832(0.020)$ & 0.002 \\
4 medium brown & $0.905(0.017)$ & $0.912(0.010)$ & 0.007 \\
5 light brown & $0.812(0.010)$ & $0.812(0.015)$ & 0.000 \\
6 red brown & $0.733(0.006)$ & $0.728(0.007)$ & -0.005 \\
7 black & $0.998(0.004)$ & $0.969(0.017)$ & -0.029 \\
8 red & $0.844(0.002)$ & $0.848(0.005)$ & 0.004 \\
9 blue & $0.710(0.013)$ & $0.715(0.021)$ & 0.005 \\
10 light orange & $0.764(0.006)$ & $0.717(0.008)$ & -0.047 \\
11 orange yellow & $0.701(0.015)$ & $0.271(0.013)$ & -0.430 \\
\hline
\end{tabular}

* Mean with standard deviation in parentheses.

Tukey's Interval is 0.024 at the $95 \%$ level of confidence.

Underline indicates no statistical difference. 
TABLE 3

DOMINANT WAVELENGTH IN nM OF PIGMENTS IN ELASTOMER AT 0 AND 900 HOURS

\begin{tabular}{lccc}
\hline \multicolumn{1}{c}{ Pigment } & 0 Hours & 900 Hours & Change \\
\hline 1 white & $565.4(3.4)^{*}$ & $582.7(0.1)$ & 17.3 \\
2 yellow & $\frac{583.0(0.1)}{591.7(0.1)}$ & $583.0(0.1)$ & 0.0 \\
3 dark buff & $\frac{603.8(0.1)}{596.3(0.1)}$ & $591.8(0.1)$ & 0.1 \\
4 medium brown & $\frac{603.7(0.3)}{60.8(0.1)}$ & $596.0(0.1)$ & -0.1 \\
5 light brown & $\underline{686.0(0.8)}$ & $604.5(0.3)$ & -0.3 \\
6 red brown & $624.8(0.2)$ & $485.9(0.8)$ & -0.3 \\
7 black & $478.9(0.4)$ & $622.4(0.3)$ & -0.1 \\
8 red & $597.0(0.2)$ & $479.6(0.3)$ & -2.4 \\
9 blue & $585.2(0.1)$ & $596.4(0.1)$ & 0.7 \\
10 light orange & $582.5(0.1)$ & -0.6 \\
11 orange yellow & & -2.7 \\
\hline
\end{tabular}

*Mean with standard deviation in parentheses.

Tukey's Interval is $0.4 \mathrm{~nm}$ at the $95 \%$ level of confidence.

Underline indicates no statistical difference.

tomer has a low contrast ratio of 0.08 when unpigmented ${ }^{4}$ and is quite translucent, changes in contrast ratio of the pigmented elastomer have the potential for changing the appearance of an appliance.

Only five pigments changed in dominant wavelength, and for four the changes were small (from -2.7 to $0.7 \mathrm{~nm}$ ). One pigment, \#1 (white), had a very large change of 17.3 $\mathrm{nm}$, moving from 565.4 to $582.7 \mathrm{~nm}$ or a shift toward the red region of the visible spectrum. When the excitation purity of a pigment is high, changes in dominant wavelength are quite visible.

The changes in excitation purity on aging were the most interesting. Only one pigment demonstrated a large increase in excitation purity, and that was \#1 (white) with a change of 0.092 . Two pigments, \#2 (yellow) and \#11 (orange yellow) had very large decreases in excitation purity of -0.669 and -0.757 , respectively. In fact, the samples had a "washed out" appearance after accelerated aging. Naturally, changes of this magnitude would ruin the color of an appliance.

From the results obtained in this study, it is possible to predict the color stability of maxillofacial pigments. Although this study was limited to one series of pigments, future studies will deal with several different types of pigments to determine the most suitable pigments for clinical use.

The analysis of the color stability of maxillofacial materials and pigments has provided useful information for the selection of

TABLE 4

EXCITATION PURITY OF PIGMENTS IN ELASTOMER AT 0 AND 900 HOURS

\begin{tabular}{lccr}
\hline \hline \multicolumn{1}{c}{ Pigment } & 0 Hours & 900 Hours & Change \\
\hline 1 white & $0.013(0.002)$ & $0.105(0.002)$ & 0.092 \\
2 yellow & $0.876(0.004)$ & $0.119(0.007)$ & -0.757 \\
3 dark buff & $0.777(0.002)$ & $0.771(0.003)$ & -0.006 \\
4 medium brown & $0.580(0.003)$ & $0.594(0.004)$ & 0.014 \\
5 light brown & $0.765(0.009)$ & $0.766(0.004)$ & 0.001 \\
6 red brown & $0.527(0.006)$ & $0.541(0.005)$ & 0.014 \\
7 black & $0.020(0.004)$ & $0.026(0.013)$ & 0.006 \\
8 red & $0.797(0.004)$ & $0.792(0.004)$ & -0.005 \\
9 blue & $0.498(0.005)$ & $0.490(0.003)$ & -0.008 \\
10 light orange & $0.914(0.002)$ & $0.890(0.006)$ & -0.024 \\
11 orange yellow & $0.903(0.001)$ & $0.234(0.008)$ & -0.669 \\
\hline
\end{tabular}

* Mean with standard deviation in parentheses.

Tukey's Interval is 0.008 at the $95 \%$ level of confidence.

Underline indicates no statistical difference. 
materials. It does not, however, adequately explain the degree of color degradation that is seen clinically. Further work will need to be carried out to analyze the stain resistance of the elastomers and the effect of handling and cleansing of the appliances by the patient.

\section{Conclusions.}

A series of eleven maxillofacial pigments was evaluated for color stability by using a reflectance spectrophotometer for color measurements and an accelerated aging chamber. Very small changes in color could be detected by this method.

All pigments demonstrated statistically significant changes in at least one parameter of color. Seven pigments showed only small color changes, and these included: \#3 (dark buff), \#4 (medium brown), \#5 (light brown), \#6 (red brown), \#7 (black), \#8 (red), and \#9 (blue). One pigment, \#10 (light orange), demonstrated a moderate color change after aging. Three pigments had very poor color stability. These were \#1 (white), \#2 (yellow), and \#11 (orange yellow).

\section{REFERENCES}

1. SWEENEY, W. T.; FISCHER, T. E.; CASTLEBERRY, D. J.; and COWPERTHWAITE, G. F.: Evaluation of improved maxillofacial prosthetic materials, J Prosth Dent, 27:297-305, 1972.

2. CANTOR, R.; WEBBER, R. L.; STROUD, L.; and RYGE, G.: Methods for evaluating prosthetic facial materials, $J$ Prosth Dent, 21: 324-332, 1969.

3. GOLDBERG, A. J.; CRAIG, R. G.; and FILISKO, F. E.: Ultraviolet light stability of external maxillofacial prosthetic materials, Abstr., $I A D R$ Program \& Abstr. of Papers, 55:B138, 1976.

4. CRAIG, R. G.; KORAN, A.; and YU, ROBERT: Color stability of elastomers for maxillofacial appliances, Abstr., IADR Program \& Abstr. of Papers, 56:A173, 1977.

5. WYSZECKI, G.; and STILES, W. S.: Color Science, New York, Wiley and Sons, Inc., 1967,628 pp.

6. Opacity of paper, D589-65 (1970): In ASTM Standards, 1975, part 20. Philadelphia, American Society for Testing \& Materials, 1975, p. 86-88.

7. DALBY, JOHN, programmer: BMD8V Analysis of variance, Ann Arbor, Statistical Research Laboratory, University of Michigan, 1968.

8. GUENTHER, W. C.: Analysis of variance, Englewood Cliffs, NJ, Prentice-Hall, 1964, 199 pp. 